\title{
Effects of Quantitative Measures on Understanding Inconsistencies in Business Rules
}

\author{
Sabine Nagel \\ University of Koblenz-Landau \\ Institute for IS Research \\ Germany \\ $\underline{\text { snagel@uni-koblenz.de }}$
}

\author{
Carl Corea \\ University of Koblenz-Landau \\ Institute for IS Research \\ Germany \\ ccorea@uni-koblenz.de
}

\author{
Patrick Delfmann \\ University of Koblenz-Landau \\ Institute for IS Research \\ Germany \\ delfmann@uni-koblenz.de
}

\begin{abstract}
Business Rules have matured to an important aspect in the development of organizations, encoding company knowledge as declarative constraints, aimed to ensure compliant business. The management of business rules is widely acknowledged as a challenging task. A problem here is a potential inconsistency of business rules, as business rules are often created collaboratively. To support companies in managing inconsistency, many works have suggested that a quantification of inconsistencies could provide valuable insights. However, the actual effects of quantitative insights in business rules management have not yet been evaluated. In this work, we present the results of an empirical experiment using eyetracking and other performance measures to analyze the effects of quantitative measures on understanding inconsistencies in business rules. Our results indicate that quantitative measures are associated with better understanding accuracy, understanding efficiency and less mental effort in business rules management.
\end{abstract}

\section{Introduction}

Business Process Management (BPM) has received widespread adaptation in the development of today's organizations [1]. Here, so-called business processes allow representing company activities and their interrelations, which helps organizations to define how the business and its employees should function in order to collaboratively pursue company goals. A central objective in BPM is to warrant efficient and compliant processes [1]-[3]. Business rules can support companies through a specification of business logic relative to business processes [2], [4]. Through this formalization of business logic, companies can ensure that processes are aligned towards company goals and regulations.
Utilizing business rules to govern business processes however strongly assumes the consistency of business rules. To clarify, the set of business rules must not contain contradictions, as this impedes a correct usage of business rules. Figure 1 shows an example of such a contradiction. Here, two contradictory business rules determining the creditworthiness of a customer are shown. Due to the inconsistency of these business rules, it is not possible to use these rules for decision making in a corresponding process.
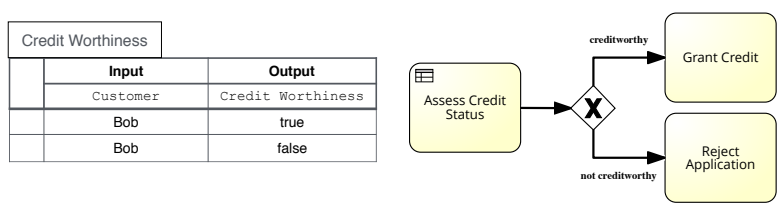

Figure 1: Exemplary process model and corresponding, inconsistent business rules

Recent research on analyzing business rules suggests that inconsistencies can be a problem in collaborative settings [5]-[11]. For instance, Batoulis and Weske [6] report on a recent case study with a large insurance company, where those authors found that $27 \%$ of analyzed business rules were erroneous. Such results emphasize the need to support companies with the capacity to manage inconsistencies in business rules [10], [11]. Next to a detection of inconsistencies, authors such as Lu et al. [10] or Sadiq and Governatori [11] suggest that companies should be provided with a quantitative analysis in the context of business intelligence, in order to promote an understanding of inconsistencies and consequently foster inconsistency resolution. The intuition is that some problems may be more severe than others and thus a quantification could provide means to assessing and prioritizing inconsistencies.

Despite the suggestion of authors in [10], [11], who explicitly point out that quantitative insights provide added-value to understanding problems in the scope of 
sustainable business rules management, this proposition has not yet been empirically evaluated.

In this report, we therefore investigate the theoretical foundation of how a quantitative analysis affects the understanding of inconsistencies in business rules. To this aim, we hypothesize the relationship between quantitative measures and understanding inconsistencies in business rules (Section 3). We present the results of an experiment showing that quantitative measures provide added-value to business rules management (Section 5). The underlying experiment design is introduced in Section 4. Our investigation is based on a preliminary discussion of inconsistency measurement in Section 2 and is concluded in Section 6.

\section{Background and Related Work}

Business rules can be distinguished into constraints for data objects (structural rules) and rules defining the principles in which business activities should be performed (behavioral rules) [2]. In this work, we focus on behavioral rules. Following Graham [2], behavioral rules are of the general form

$$
\text { If } I_{1}, \ldots, I_{n} \rightarrow O
$$

where $I_{l}, \ldots, I_{n}$ is a premise comprising certain inputs, and $O$ is the outcome of the rule which can be concluded if the premise is satisfied. Numerous standards to model business rules following this if/then structure have been introduced and adopted in practice [4]. As a design choice, we consider the Decision Model and Notation (DMN) ${ }^{1}$ as a standard to represent business rules, as this can be seen as a current industry standard complementary to the Business Process Model and Notation (BPMN) standard for modeling business processes [6], [8].

DMN allows modeling business rules in decision tables. An example is shown in Figure 2.

\begin{tabular}{|c|c|c|}
\hline \multicolumn{2}{|c|}{ Credit Worthiness } \\
\multicolumn{2}{c|}{} \\
\hline & Input & Output \\
\cline { 2 - 3 } & Account Balance & Credit Worthiness \\
\hline 1 & $<=10.000 €$ & true \\
\hline 2 & $10.000 €$ & false \\
\hline 3 & $>=10.000 €$ & true \\
\hline
\end{tabular}

Figure 2: Exemplary DMN table containing inconsistencies

\footnotetext{
${ }^{1}$ https://www.omg.org/spec/DMN/About-DMN/
}

The columns in decision tables are used to denote the in- and outputs of a rule. The rows of decision tables relate to individual business rules. In Figure 2, one can observe three business rules modeling the creditworthiness of customers based on the input of account balance. Rule 1 in Figure 2 can be read as ,,if the Account Balance is $<=10.000 €$, then the customer is credit-worthy". The other two rules can be interpreted analogously. As can be seen, the set of business rules is inconsistent, due to an overlap and contradictory conclusions of rule 2 with respect to the other rules.

Following Zhang \& Norman [12] and Sadiq \& Governatori [11], the modeling of business rules in standards such as DMN is performed in an incremental and collaborative process. Diverging views or understandings on the same domain can yield contradicting rules, making the set of business rules inconsistent.

There have been some works towards a detection of inconsistencies in business rules [6], [8], [9], [13]. One approach of inconsistency detection is to consider all business rules as a set, denoted as a rule base. We define inconsistency of a rule base $B$ as logical inconsistency, i.e. there is support for contradictory output , $O$ “ and ,not $O$ “ at the same time. This settheoretic view allows defining minimal inconsistent subsets MIS of $B$ via

$$
\begin{aligned}
\operatorname{MIS}(B)= & \left\{B^{\prime} \subseteq B \mid B^{\prime}\right. \text { is inconsistent and minimal } \\
& \text { in terms of set inclusion }\} .
\end{aligned}
$$

This definition of minimal inconsistent subsets can be applied to find inconsistencies in a rule base $B$. For example, the minimal inconsistent subsets for the rule base in Figure 2 are shown in Figure 3 Here, two minimal inconsistent subsets can be identified, denoted as $\mathrm{MIS}_{1}$ and $\mathrm{MIS}_{2}$.

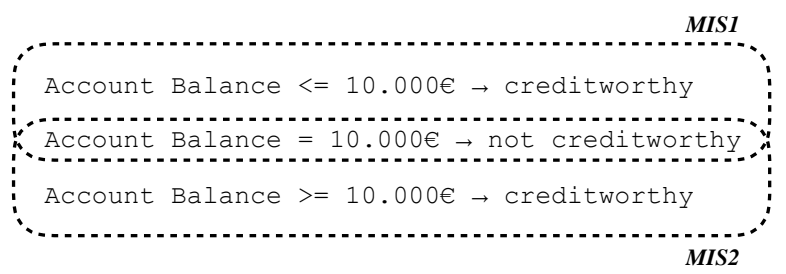

\section{Figure 3: Minimal inconsistent subsets for Figure 2}

Next to this detection, there is a broad consensus that a quantitative inconsistency analysis in the context of business intelligence could provide added-value to the development and management of business rules [9]-[11], [13]. A scientific field concerned with such a quantitative analysis is the field of inconsistency measurement [14]. Here, a central object of study are 
so-called inconsistency measures, which allow assigning a numerical value to (business) rules, with the informal meaning that a higher value reflects a higher degree of inconsistency [15]. Inconsistency measures subsequently provide the technical means for quantitative analysis of business rule inconsistencies. Let $\boldsymbol{B}$ be the set of all business rule bases, and $\boldsymbol{R}$ the set of all rules that appear in the individual rule bases $\in \boldsymbol{B}$. Then, an inconsistency measure to assess the degree of inconsistency for individual rules is a function

$$
I: \boldsymbol{B} \times \boldsymbol{R} \rightarrow[0, \infty)
$$

which assigns a non-negative value to a combination of a rule base and a specific rule. This measure can thus assess the culpability that this rule represents with respect to the inconsistency of a rule base. An example is the so-called $C_{\#}$ measure [16] which assesses the culpability of a rule $r$ for a rule base $B$, via

$$
C_{\#}(B, r)=|\{M \in M I S(B) \mid r \in M\}| \text {. }
$$

This measure counts the number of minimal inconsistent subsets that a rule $r$ belongs to. Applying this measure to the rule base in Figure 3 results in the following quantification:

$$
\begin{aligned}
& C_{\#}(B, \text { Rule } 1)=2 \\
& C_{\#}(B, \text { Rule } 2)=1 \\
& C_{\#}(B, \text { Rule } 3)=1
\end{aligned}
$$

Following Thimm [15], a quantification by inconsistency measures such as the $C_{\#}$ measure allows to assess the severity of inconsistency for individual rules. This allows ranking individual rules by their degree of inconsistency, where the rules are sorted by the respective value as quantified by the inconsistency measure. Given the rule base in Figure 3 and the corresponding quantification in (5), Rule 1 consequently has the highest degree of inconsistency, i.e. the highest amount of blame in the context of the overall inconsistency.

This ranking can be displayed to the user as shown in Figure 4, providing quantitative insights as a basis for an informed inconsistency resolution strategy.

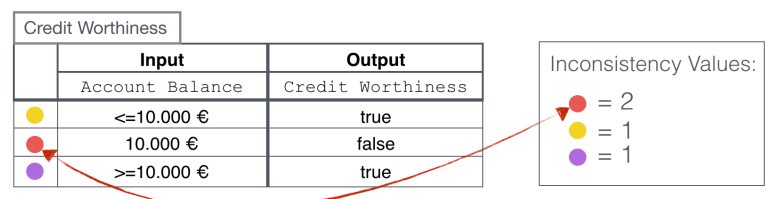

Figure 4: DMN table with inconsistency values (example)
For a specific quantitative measure, we use inconsistency measures to assess the severity of inconsistency for individual business rules. In the following, we denote the degree of inconsistency for a rule as its inconsistency value.

In theory, an application of quantitative measures for analyzing business rule bases seems intuitive. Yet, the added-value of quantitative business intelligence insights to understanding inconsistencies in business rules has not been evaluated. In the following, we present the results of our experiment empirically assessing these effects.

\section{Research Aim}

In this work, we follow an Experimental Research methodology as described by Neuman [17]. Experimental Research focuses on causal relations, allowing to isolate and target the impacts of causal variables. Experimental Research is highly suitable for research aimed at investigating the effects of independent variables. To this aim, the researcher controls a condition in which events occur, manipulates the independent variable and analyses the causal effects that occur based on this manipulation. As the aim of this work is to evaluate the effect of quantitative measures, we see this methodology as highly appropriate, as providing a quantification can be seen as an independent variable and thus the causal effects of providing, resp. not providing, a quantification can be assessed.

Following [17], Experimental Research comprises the steps of formulating a research question, develop hypotheses based on an independent variable, performing the experiment (i.e. data collection), and analyzing the results.

Aligned with the aim of our work as motivated in our introduction, we derive the following research question:

RQ: How do quantitative measures affect the understanding of inconsistencies in business rules?

In order to investigate the causal effect of quantitative measures on the understanding of inconsistencies in business rules, we developed three hypotheses.

The first aim was to investigate the effect of quantitative measures on understanding accuracy, which means how well a user can understand inconsistency related problems in business rules: 
Hypothesis 1: Providing a quantification of inconsistency in business rules is associated with better understanding accuracy compared to manual analysis. Following [11], the quantitative insights provided by inconsistency analysis increase the efficiency in which modelers can understand problems in business rule bases. To evaluate this proposition, we investigated understanding efficiency, which relates to how much time it takes a participant to understand the scenario and answer corresponding questions.

Hypothesis 2: Providing a quantification of inconsistency in business rules is associated with better understanding efficiency compared to manual analysis.

Last, we investigated mental effort, as quantitative insights could lower cognitive skills needed for understanding inconsistencies in rule bases:

Hypothesis 3: Providing a quantification of inconsistency in business rules is associated with less mental effort needed for problem understanding compared to manual analysis.

The dimensions of our hypotheses, i.e. understanding accuracy, efficiency, and less mental effort, are based on the experiment design in [18]. To test these hypotheses, we performed controlled experiments with participants. The following section provides insights into our experiment design, as well as the measures used to verify our hypotheses against the data collected in our experiments.

\section{Experiment}

In this section, we introduce the experiment ${ }^{2}$ we conducted as part of this research, including the experiment design, structure, measurements, settings, and participants.

\subsection{Experiment Design}

In order to test our hypotheses, we conducted an experiment where participants were confronted with questions and scenarios regarding inconsistencies in business rules. The experiment was designed as a single-factor experiment, which is especially suitable to analyze the effects of a single factor on a common response variable following Reijers et al. [19]. The considered factor is the use of quantitative measures, with factor levels "present" and "absent". We used two groups of participants, which we each tested in two separated domains. A domain is defined as a test run, which comprises a block of comprehension questions. Each test run was performed with two different factor configurations, one with and the other one without access to quantitative measures.

The experiment was designed as a balanced single factor experiment with repeated measurement, based on an experiment design from [19]. This means that all participants used all factor levels, which lead to every subject generating data for both domains with respective factor levels. This approach enables the collection of more precise and powerful data as the same number of participants generates twice as much data [20]. In addition, each scenario was only shown once to each participant, to mitigate learning effects.

The design of our experiment is illustrated in Figure 5. In the first run, Group 1 was exposed to tasks 1-4 with access to inconsistency values, while Group 2 started without a respective quantitative insight. In the second run the situation was inverted, i.e. the first group had no access to inconsistency values to work on tasks 5-8, whereas Group 2 was provided with inconsistency values.

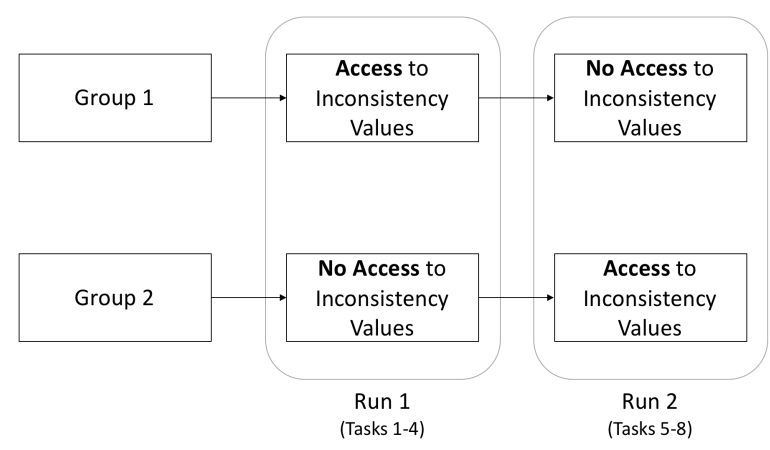

Figure 5: Experiment design

As each participant was tested for all domains and factor levels, the generalizability of the results was increased. A potential distortion due to learning effects was counterbalanced across groups since the order of factor levels was reversed between groups.

The questions were formulated in English in order to enable participants with different native languages to participate in the experiment, as well as to allow for better comprehension in the scope of reproducible research.

Figure 6 shows an exemplary scenario from the second domain.

\footnotetext{
${ }^{2}$ The experiment can be downloaded from: https://cloud.unikoblenz-landau.de/s/tJ5C8Ky2PEoCt89
} 


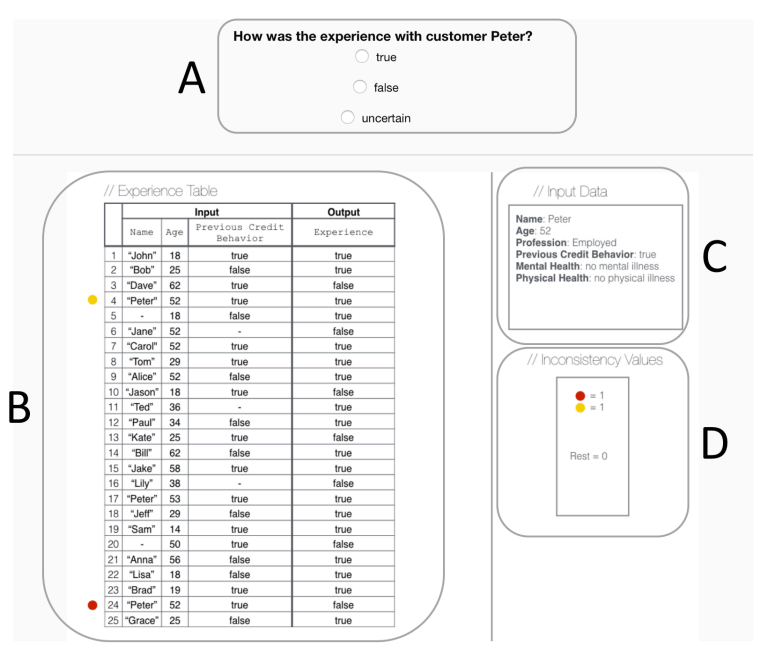

Figure 6: Exemplary scenario

Every task was divided into four areas. At the top, a (single-choice) question including relevant response options is displayed (A). Below the question, the scenario is shown, which is divided into three areas. On the left, one or more DMN tables (B) are shown. The tables can either be independent or connected to each other. In some cases, the participant is provided with input data (C) that can be used to identify the relevant rules in the DMN table. If a participant has access to inconsistency values, they are shown in the corresponding box (D). Otherwise, the box is empty.

\subsection{Experiment Structure}

All participants were shown a general introduction to the experiment including a tutorial before being exposed to the scenarios. The tutorial covered the basics of decision management and DMN tables. Additionally, the quantitative measures used in the experiment were introduced.

Each run consisted of four different tasks, containing a scenario and a corresponding question. The scenarios were all independent from each other and were designed to cover common types of inconsistencies in the area of decision management. While some scenarios only contained a single DMN table, others covered inconsistencies across multiple tables.

Across both domains there were three different types of questions:

- Content-related questions, that could be answered with true, false or uncertain (e.g. "Is Dave contractually capable?"). Here, the answer uncertain was correct for questions where no conclusion could be inferred due to an inconsistency of rules.
- Questions that asked for specific rules with the highest/lowest amount of inconsistencies and that could be answered with a particular rule (e.g. "Which rule is in conflict with the highest number of other rules?")

- Questions that asked for the number of inconsistencies of a particular rule (e.g. "How many rules contradict rule 1 ?")

\subsection{Measurements}

In our experiment, we distinguish between three types of measurements, referred to as response variables. To measure understanding accuracy, we use the percentage of correctly answered comprehension questions. Next, understanding efficiency was measured by tracking the time from the point that the first question of a run is displayed to the point that the participants selected the answer to the last question of a run. Last, mental effort was measured using eyefixation duration, which is the period of time where the eyes remain still and focused on a single location. During the eye saccade or movement, vision is suppressed. New information can only be acquired during fixation [21]. The longer the fixation duration, the longer it takes for humans to comprehend respective information. Thus, eye-fixation duration can be used as an objective measure for mental effort [22]. In addition to this objective measure, the participants were asked which run they found easier as a measure of perception of required mental effort.

\subsection{Settings}

The tasks were implemented as HTML-files and in combination with the introductory slides - added to the corresponding run using the eye-tracking software Tobii Studio. A pre-test was used to verify that the texts and tables were clearly visible from a distance of over $60 \mathrm{~cm}$, which is the distance required by the eyetracker (cf. Section 4.5 for details on the pre-test).

The screen was divided into four sections (see Figure 6, section 4.1). The questions were shown at the top and the corresponding DMN tables, input data and inconsistency values were shown below. All sections did neither require nor allow scrolling or zooming. We used Tobii X60, an eye tracker with a 22-inch screen of a resolution of $1680 \times 1050$. The experiment was set in an IT lab at the University of Koblenz-Landau, Germany. The blinds in front of the windows were closed and the lights on the ceiling were the only light source. The settings were the same for all participants. 


\subsection{Participants}

In advance of the actual experiment, we conducted a pre-test with seven Ph.D. students. The main goal of the pre-test was to ensure understandability, readability and the overall usability of the experiment. After using the results of the pre-test to refine the introductory slides and the resolution and size of the scenarios, the experiment was conducted with 37 Bachelor and Master Students from the Faculty of Computer Science at the University of Koblenz-Landau. Study programs at this faculty range from computer sciences to economic sciences including interdisciplinary courses such as Business Process Management. The experiment required no prior knowledge as all relevant concepts were introduced in the tutorial. However, we took into consideration that all participants came from a business informatics background as they pursue an IT-related degree. The students were randomly assigned to two groups and they participated voluntarily, so no incentive was offered.

\section{Results}

After data collection through our experiment, we assessed the participant data with the respective measures described in Section 4.3 to evaluate our hypotheses.

First, we checked whether each dependent variable was normally distributed. To this aim, we assumed the data to be normally distributed if the standardized skewness and standardized kurtosis are within the range $[-2,+2]$, following [19]. If the results of both groups in one run were normally distributed, we used Levene's test at a significance level of 0.05 to check whether the variance of both samples was equal. Given equal variance, we applied an independent-sample ttest. If the data was not normally distributed, we ran the Mann-Whitney $U$ test, which can indicate a significant difference between two sample medians of not normally distributed samples [23]. For both the ttest and the Mann-Whitney $U$ test, we assumed the commonly used significance level of 0.05 .

\subsection{Understanding Accuracy}

Figure 7 depicts an overview of test results for the understanding accuracy measurement. The $\mathrm{x}$-axis groups the individual task results. The y-axis shows the mean of the number of correctly answered questions across all participants. In five out of eight tasks, the percentage of correct answers differed by at least $40 \%$, suggesting higher understanding accuracy for test runs with access to inconsistency values.

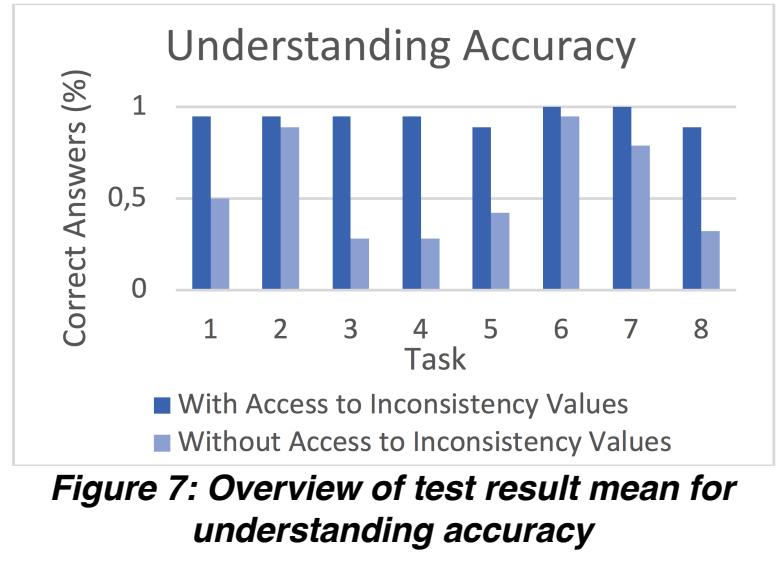

Both standardized skewness and standardized kurtosis were not within the range $[-2,+2]$. Also, due to the fact that we asked four questions per run, there could only be five distinct results for the percentage of correct answers $(0,0.25,0.5,0.75,1)$, meaning the data was not continuous. Hence, we could not apply other tests to check normal distribution such as the Kolmogorov-Smirnov test, as these tests are not applicable for discrete data. Therefore, the values could not be assumed to be normally distributed. Consequently, we ran the Mann-Whitney U test. The results are shown in Table 1.

Table 1: Test of Hypothesis 1 (understanding accuracy)

\begin{tabular}{|c|c|c|c|c|}
\hline & \multicolumn{2}{|c|}{ Run 1 } & \multicolumn{2}{c|}{ Run 2 } \\
\hline Group & 1 & 2 & 1 & 2 \\
\hline N & 19 & 18 & 19 & 18 \\
\hline Mean & 0.95 & 0.49 & 0.62 & 0.94 \\
\hline Standard & 0.13 & 0.21 & 0.19 & 0.16 \\
Deviation & \multicolumn{2}{|c|}{} & \multicolumn{2}{|c|}{$<0.0001$} \\
\hline p (1-tailed) & \multicolumn{2}{|c|}{$<0.0001$} & \multicolumn{2}{c|}{} \\
\hline
\end{tabular}

As both p-values are statistically significant, the understanding accuracy was correlated with the access to inconsistency measures in both runs, which supports Hypothesis 1.

Conclusion 1: Quantitative measures for business rule inconsistencies are associated with an improved understanding accuracy.

\subsection{Understanding Efficiency}

Figure 8 shows an overview of test results for the understanding efficiency measurement. The results for the individual tasks are grouped along the $\mathrm{x}$-axis. The $y$-axis indicates the time needed for answering a task in seconds. As can be seen, participants with access to 
inconsistency values had a lower completion time for all tasks, indicating higher understanding efficiency.

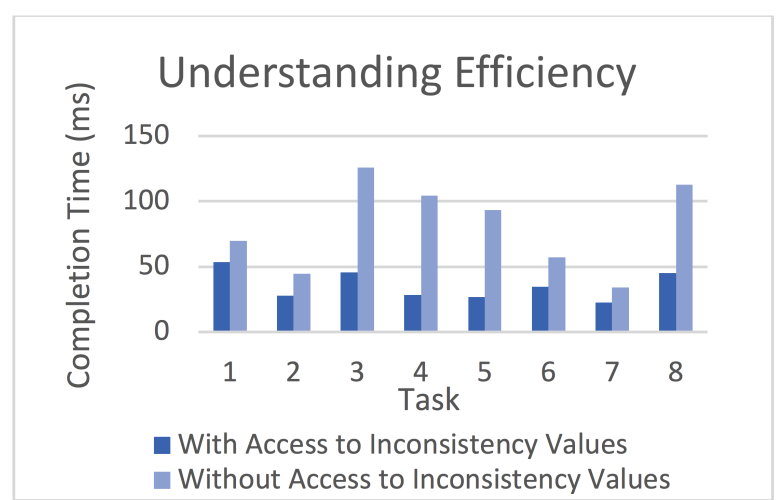

Figure 8: Overview of test result mean for time taken for task completion

The time the participants spent to answer the questions was normally distributed and both samples had equal variances ( $p$ values of Levene's test were 0.087 and 0.101 , respectively). We consequently ran independent-sample t-tests between the two groups for each run. Table 2 shows the results of this test.

Table 2: Test of Hypothesis 2 (understanding efficiency)

\begin{tabular}{|c|c|c|c|c|}
\hline & \multicolumn{2}{|c|}{ Run 1 } & \multicolumn{2}{c|}{ Run 2 } \\
\hline Group & 1 & 2 & 1 & 2 \\
\hline N & 19 & 18 & 19 & 18 \\
\hline Mean & 38.89 & 86.08 & 74.47 & 32.32 \\
\hline $\begin{array}{c}\text { Standard } \\
\text { Deviation }\end{array}$ & 23.72 & 32.25 & 31.45 & 15.25 \\
\hline $\mathrm{t}$ & \multicolumn{2}{|c|}{-4.948} & \multicolumn{2}{c|}{5.002} \\
\hline $\mathrm{p}$ (1-tailed) & \multicolumn{2}{|c|}{$<0.0001$} & \multicolumn{2}{c|}{$<0.0001$} \\
\hline
\end{tabular}

The p-values are statistically significant. Thus, the understanding efficiency was correlated with the access of inconsistency measures in both runs, which supports Hypothesis 2.

Conclusion 2: Quantitative measures for business rule inconsistencies are associated with an improved understanding efficiency.

\subsection{Mental Effort}

In order to measure mental effort, we used the eyefixation duration. Here, we encountered the problem that the eye-tracking did not work for all 37 participants. Even though we cannot provide a verifiable technical explanation for this, we noticed that this phenomenon exclusively affected participants with glasses. To clarify, this only affected a fraction of participants with glasses. Based on this observation and information provided by the manufacturer of the eye-tracker [24], we assume that the condition of specific glasses and their reflections could be responsible for this. As the eye-tracking results were only needed as a measure for Hypothesis 3 , we decided to still include the data of these participants towards evaluating understanding accuracy and efficiency. Accordingly, $\mathrm{N}$ was 30 for the eye-tracking measurement.

Figure 9 displays an overview of test results for the objective mental effort measurement. The respective task results are grouped by task on the x-axis. The average eye-fixation duration is shown on the y-axis. For all tasks, participants who had access to inconsistency values displayed a lower fixation duration, meaning that the time for comprehending information during a cognitive load was significantly lower. Following [21], this suggests lower mental effort during task completion.

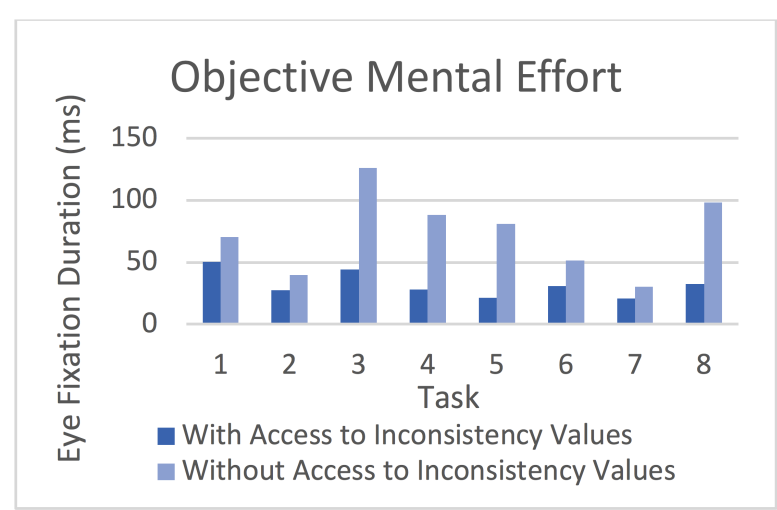

\section{Figure 9: Overview of test result mean for} objective mental effort

The eye-fixation durations were normally distributed, and both samples had equal variances $(\mathrm{p}$ values of Levene's test were 0.082 and 0.193 , respectively), so we ran independent-sample t-tests between the two groups for each run.

Table 3: Test of Hypothesis 3 (objective mental effort)

\begin{tabular}{|c|c|c|c|c|}
\hline & \multicolumn{2}{|c|}{ Run 1 } & \multicolumn{2}{c|}{ Run 2 } \\
\hline Group & 1 & 2 & 1 & 2 \\
\hline N & 15 & 15 & 15 & 15 \\
\hline Mean & 37.38 & 81.05 & 65.18 & 26.46 \\
\hline Standard & 23.27 & 33.46 & 23.39 & 14.54 \\
Deviation & \multicolumn{2}{|c|}{} & & \multicolumn{2}{|c|}{5.261} \\
\hline t & \multicolumn{2}{|c|}{-4.009} & \multicolumn{2}{c|}{$<0.0001$} \\
\hline p (1-tailed) & \multicolumn{2}{|c|}{0.0002} & \multicolumn{2}{c}{} \\
\hline
\end{tabular}


The p-values are both statistically significant for the eye-fixation duration, which suggests less mental effort being correlated with the access to inconsistency measures in both runs, supporting Hypothesis 3 .

In addition to the objective mental effort, we also asked the participants which run, i.e. the run with or without access to inconsistency values, they found easier or whether they perceived them as equally easy. 36 out of the 37 participants associated the run with access to inconsistency measures with less mental effort and only one person found the run without inconsistency measures easier.

Conclusion 3: Quantitative measures for business rule inconsistencies are associated with reduced mental effort required for understanding and interpreting inconsistencies in business rules.

Figure 10 and Figure 11 show heat maps for a scenario from the first domain without and with access to inconsistency values. Both figures show data from all participants of the corresponding run. Heatmaps display the focus of visual attention and how visual gaze is distributed. When comparing Figure 10 and Figure 11 it is observable that the participants without access to inconsistency values spent a lot more time and effort analyzing the four columns of the table. In Figure 10, participants had a large fixation-duration over the entirety of columns in the DMN tables.

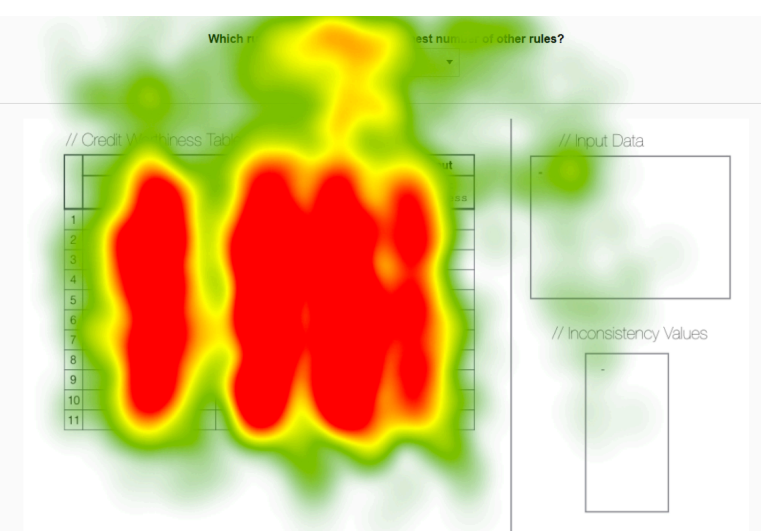

Figure 10: Heat map for task 3 without access
to inconsistency values

On the contrary, the participants with access to inconsistency values, however, only briefly looked at the different columns after checking the provided inconsistency values. It is visible that participants who had quantitative insight were able to ignore irrelevant parts of the rule base, allowing for a more efficient task completion. Also, Figure 11 shows that participants actively looked at and used the provided quantitative ranking.

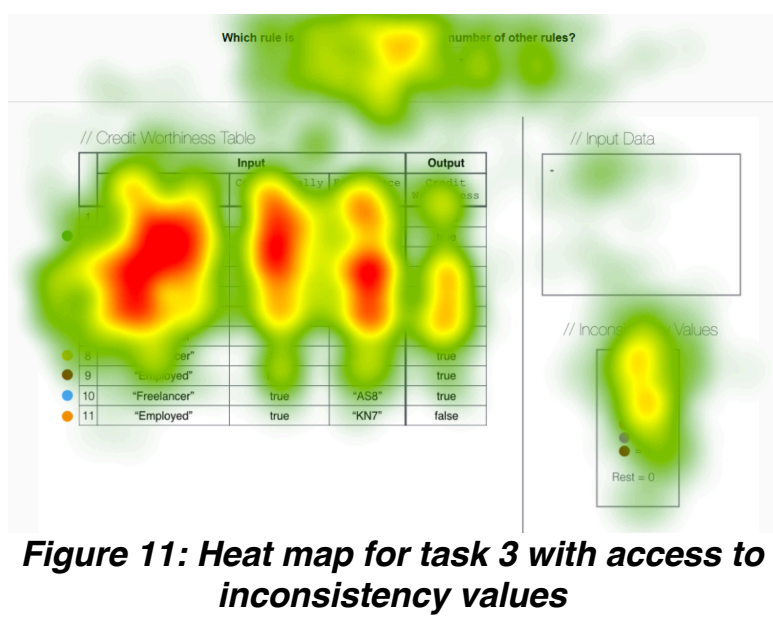

\section{Discussion \& Conclusion}

In this paper, we presented the results of an experiment investigating the effects of quantitative measures on understanding inconsistencies in business rules.

To verify our hypotheses, our focus was on three measurements: understanding accuracy, understanding efficiency, and mental effort measured via the percentage of correct answers to comprehension questions, the time needed for solving a given task and eye-fixation duration, respectively. The data was accumulated in empirical experiment research.

Due to the experimental nature of our research, potential limitations should be considered.

Our results are based on an experiment with a total number of 37 participants, which could affect the external validity. To put our sample size into perspective we compare our participant size to the number of participants in other related studies. The identified studies include [22] (23 participants), [19] (28 participants), [25] (28 participants) and [18] (50 participants), indicating that our sample size is comparable to those of mentioned works. Furthermore, the results of our t-tests have very low p-values (most below 0.0001), which already indicates an extremely significant effect in the analyzed sample.

It is possible that a change in the structure of the different scenarios or the order of questions might have an effect on the experiment results. Also, English being our language of choice could have had an impact on the participants' performance, as English was not the native language of our subjects and the comprehension of the scenarios therefore depends on the individual language skills. However, we want to point out that the 
situation was the same for all participants in order to enable internal validity.

The fact that our group of participants only consisted of students as opposed to domain experts, could have an impact on external validity, as the scenarios used in this experiment do not necessarily reflect those that occur in practice. However, since these scenarios represent typical inconsistencies in business rule bases, an application of our results to other domains seems plausible.

Our results support all three hypotheses, which were introduced in section 3. This indicates that quantitative measures are associated with better understanding accuracy, understanding efficiency and less mental effort in business rules management. Due to the applied experimental research methodology, our conclusions are based on the tested group of participants. To conclude, quantitative insights provide added-value to business intelligence by supporting companies in understanding inconsistencies in business rules. Following the suggestions by [10], [11], this work is the first to empirically show these effects.

We identify several possibilities for future work. The inconsistency quantification in our experiments was based on the C\# measure. Future work could investigate how other inconsistency measures impact our experiment results. Additionally, the impact of different visualization techniques could also be evaluated.

Based on our results, we can identify several implications for practitioners. First, our results show positive effects of quantitative measures. This validates the findings by [10]. While there has been a plethora of work geared towards automatisms for the qualitative analysis of rule bases (i.e. a detection of errors), automated quantitative analysis has received far less attention. Efforts should therefore be directed towards incorporating quantitative measures in Business Rules Management. This quantification provides a clearly articulated impact assessment of compliance risks and inconsistencies, which can be used as a basis for an informed decision regarding a prioritization of problems and a time-efficient inconsistency resolution by domain experts.

Last, as suggested by [18], we utilized neurophysiological measurement for industry-related studies. Current devices are becoming more attainable and less intrusive while allowing for in-depth analysis of human behavior in interaction with technology. This form of measurement could provide new opportunities for companies to understand the added-value of BI solutions.

Future research should be geared towards implementing quantitative insights in business intelligence, fostering inconsistency resolution and thus a correct decision-making, and sustainable development of organizations.

\section{Acknowledgements}

We thank Wei Wang, Marta Indulska, Shazia Sadiq and Barbara Weber for insights and inspiration to our experiment design.

\section{References}

[1] M. Weske, "Business Process Management Architectures," in Business Process Management, Springer, Berlin, Heidelberg, 2012, pp. 333-371.

[2] I. Graham, Business rules management and service oriented architecture: a pattern language. Chichester, England; Hoboken, NJ: John Wiley, 2006.

[3] W. M. P. van der Aalst, M. L. Rosa, and F. M. Santoro, "Business Process Management," Bus. Inf. Syst. Eng., vol. 58, no. 1, pp. 1-6, Feb. 2016.

[4] F. Imgrund, M. Malorny, and C. Janiesch, "Eine Literaturanalyse zur Integration von Business Rules und Business Process Management," in Internationale Tagung der Wirtschaftsinformatik, St.Gallen, 2017, p. 15.

[5] K. Batoulis and A. Nesterenko, "Decision Management in the Insurance Industry: Standards and Tools," in BPM 2017 Industry Track co-located with the 15th International Conference on Business Process Management, Cham, 2017, p. 11.

[6] K. Batoulis and M. Weske, "Soundness of DecisionAware Business Processes," in Business Process Management Forum, Springer, Cham, 2017, pp. 106124.

[7] D. Calvanese, M. Dumas, F. M. Maggi, and M. Montali, "Semantic DMN: Formalizing Decision Models with Domain Knowledge," in Rules and Reasoning, vol. 10364, S. Costantini, E. Franconi, W. Van Woensel, R. Kontchakov, F. Sadri, and D. Roman, Eds. Cham: Springer International Publishing, 2017, pp. 70-86.

[8] D. Calvanese, M. Dumas, Ü. Laurson, F. M. Maggi, M. Montali, and I. Teinemaa, "Semantics and Analysis of DMN Decision Tables," in International Conference on Business Process Management, 2016.

[9] C. Di Ciccio, F. M. Maggi, M. Montali, and J. Mendling, "Resolving inconsistencies and redundancies in declarative process models," Inf. Syst., vol. 64, pp. 425-446, Mar. 2017.

[10] R. Lu, S. Sadiq, and G. Governatori, "Measurement of Compliance Distance in Business Processes," Inf. Syst. Manag., vol. 25, no. 4, pp. 344-355, Oct. 2008.

[11] S. Sadiq and G. Governatori, "Managing Regulatory Compliance in Business Processes," in Handbook on Business Process Management, vol. 2, 2015, p. 23.

[12] J. Zhang and D. A. Norman, "Representations in distributed cognitive tasks," Cogn. Sci., vol. 18, no. 1, pp. 87-122, Jan. 1994.

[13] J. Grant and A. Hunter, "Measuring Consistency Gain and Information Loss in Stepwise Inconsistency 
Resolution," Symb. Quant. Approaches Reason. Uncertain., vol. 6717, no. 1, pp. 362-373, 2011.

[14] J. Grant and M. V. Martinez, Measuring Inconsistency in Information. College Publications, 2018.

[15] M. Thimm, "On the Compliance of Rationality Postulates for Inconsistency Measures: A More or Less Complete Picture," KI - Künstl. Intell., vol. 31, no. 1, pp. 31-39, Mar. 2017.

[16] A. Hunter and S. Konieczny, "Measuring Inconsistency through Minimal Inconsistent Sets," Knowl. Represent. Reason. KR, vol. 8, no. 1, p. 9, 2008.

[17] W. L. Neuman, Basics of social research: Qualitative and quantitative approaches. PearsonEducation,Inc', 2007.

[18] W. Wang, M. Indulska, S. Sadiq, and B. Weber, "Effect of Linked Rules on Business Process Model Understanding," in International Conference on Business Process Management, Cham, 2017, vol. 10445, pp. 200-215.

[19] H. A. Reijers, J. Mendling, and R. M. Dijkman, "Human and automatic modularizations of process models to enhance their comprehension," Inf. Syst., vol. 36, no. 5, pp. 881-897, Jul. 2011.
[20] G. Charness, U. Gneezy, and M. A. Kuhn, "Experimental methods: Between-subject and withinsubject design," J. Econ. Behav. Organ., vol. 81, no. 1, pp. 1-8, Jan. 2012.

[21] K. Rayner, "Eye Movements in Reading and Information Processing: 20 Years of Research," Psychol. Bull., vol. 124, no. 3, pp. 372-422, 1998.

[22] R. N. Meghanathan, C. van Leeuwen, and A. R. Nikolaev, "Fixation duration surpasses pupil size as a measure of memory load in free viewing," Front. Hum. Neurosci., vol. 8, Jan. 2015.

[23] D. J. Sheskin, Handbook of Parametric and Nonparametric Statistical Procedures: Third Edition. CRC Press, 2003.

[24] Tobii, "Glasses, lenses and eye surgery," Tobii Eye Tracking Support. [Online]. Available: http://help.tobii.com/hc/en-us/articles/210249865Glasses-lenses-and-eye-surgery. [Accessed: 12-Jun2018].

[25] F. A. Haji, D. Rojas, R. Childs, S. de Ribaupierre, and A. Dubrowski, "Measuring cognitive load: performance, mental effort and simulation task complexity," Med. Educ., vol. 49, no. 8, pp. 815-827, 2015. 